\title{
Analyse de la zone d'endommagement et de la fissuration fragile sous chargement cyclique dans les tubes de gaz en polyéthylène
}

\author{
Rabia Khelif ${ }^{1,2, a}$, Nassereddine Zeghib, Alaa Chateauneuf ${ }^{3}$ et Kamel Chaoui ${ }^{2}$ \\ 1 LaMI - UBP \& IFMA, Campus de Clermont-Ferrand, BP 265, 63175 Aubière Cedex, France \\ ${ }^{2}$ LR3MI, Département de Mécanique, Faculté des Sciences de l'Ingénieur, Université Badji Mokhtar, BP 12, Annaba 23000, \\ Algérie \\ 3 LGC - UBP, Complexe Universitaire des Cézeaux, BP 206, 63174 Aubière Cedex, France
}

Reçu le 15 mars 2007, accepté le 7 juin 2007

\begin{abstract}
Résumé - Les tubes en plastiques utilisés pour le transport d'eau et de gaz continuent à être le sujet de beaucoup d'études qui traitent divers aspects de comportement de matériaux. Des statistiques récentes indiquent que plus de $90 \%$ de systèmes de distribution de gaz nouvellement installés dans le monde entier sont exclusivement construits en polyéthylène $(\mathrm{PE})$ en raison de sa facilité d'installation et de coûts relativement bas. Les essais à charge constante montrent deux mécanismes généraux de propagation de fissure : une rupture ductile qui est dominée par des déformations homogènes à grande échelle dans le volume et une rupture fragile qui commence aux points de concentration des contraintes. Ce travail vise à étudier la transition fragile-ductile de fatigue dans les tubes de polyéthylène et la caractérisation de la zone d'endommagement associée. La méthode proposée est basée sur la mesure de deux paramètres de fatigue : la vitesse de propagation de la fissure, obtenue à différents niveaux de charge et le taux de travail irréversible qui est calculé à partir des boucles instantanées d'hystéréisis. Les corrélations obtenues, pour des charges maximales de fatigue entre $20 \%$ et $35 \%$ de la contrainte au seuil d'écoulement, donnent des taux de restitution d'énergies critiques moyens de $211 \mathrm{~J} . \mathrm{m}^{-2}$ et de $695 \mathrm{~J}^{-\mathrm{m}^{-2}}$ respectivement pour des régimes fragile et ductile.
\end{abstract}

Mots clés : Tubes en polyéthylène / fissuration sous fatigue / transition fragile-ductile

\begin{abstract}
Damage zone analysis and brittle cracking under cyclic loading in polyethylene gas pipes. Plastic pipes used for water and gas transmission continue to be the subject of many studies that treat various aspects of material's behaviour. Recent statistics indicate that more than $90 \%$ of gas distribution systems lately installed in the whole world are made exclusively of polyethylene (PE) because of its ease of installation and relatively low costs. Constant load tests show two general mechanisms of crack propagation: a ductile rupture, which is dominated by homogeneous deformations on a large scale in the volume and a brittle fracture that starts at stress concentration points. This work aims at studying the brittle-to-ductile transition from fatigue in polyethylene tubes and the characterization of the associated damage zone. The suggested method is based on the measurements of two fatigue parameters: the crack growth rate, obtained at various load levels and the irreversible work spent on damage and crack growth, which is calculated using instantaneous hysterisis loops. The obtained correlations, for maximum fatigue loads between $20 \%$ and $35 \%$ of the yield stress, give average critical energy release rates of $211 \mathrm{~J}^{-\mathrm{m}^{-2}}$ and $695 \mathrm{~J}^{-2}$ respectively for brittle and ductile regimes.
\end{abstract}

Key words: Polyethylene pipe / fatigue crack / brittle to ductile transition

\section{Introduction}

Les réseaux de distribution de gaz naturel et d'eau dans les villes sont fondamentalement construits en

\footnotetext{
a Auteur correspondant : rabia.khelif@ifma.fr
}

matières plastiques avec des diamètres dépassant les $250 \mathrm{~mm}$ à des pressions moyennes de 4 bars. L'intérêt accordé à ces polymères est traduit par le nombre important d'études qui traitent divers aspects en termes de durée de vie [1], de caractérisation mécanique et relation structurale [2], de modes de chargement [3], de contraintes 


\section{Nomenclature}

\begin{tabular}{|ll|}
\hline$a$ & Longueur de la fissure en mm \\
$C$ & Paramètre de capacité de charge \\
$P$ & Énergie potentielle en J \\
$W_{i}$ & Travail irréversible d'endommagement en $\mathrm{J}$ \\
$N$ & Nombre de cycles en cycles \\
$G_{\mathrm{C}}$ & Taux de restitution d'énergie critique en ${\mathrm{J} . \mathrm{m}^{-2}}^{-2}$ \\
$J_{1}$ & Taux de restitution d'énergie (ERR) mesurés expérimentalement en ${\mathrm{J} . \mathrm{m}^{-2}}_{n}$ \\
$\sigma$ & Indice d'évolution de dommage \\
$t_{0}$ & Contrainte appliquée en MPa \\
$t_{\mathrm{f}}$ & Temps de rupture en heure \\
$\frac{1}{t_{0}} \frac{\mathrm{d} a}{\mathrm{~d} N}$ & Taux de progression de la fissure en $\mathrm{m}^{2}$. cycle $^{-1}$ \\
$\sigma_{y}$ & Contrainte limite élastique en MPa \\
$R$ & rapport des charges (mix/max) \\
\hline
\end{tabular}

résiduelles [4], de mécanismes de défaillance [5] et d'effets de l'environnement [6].

Le dimensionnement des tubes thermoplastiques est réalisé par la méthode (Rate Process Method for Projecting Performance of Polyethylene Piping Components) qui est consignée dans les normes ASTM D-2837 et D-2513. Le calcul est basé sur une équation regroupant 3 coefficients liant le temps de rupture, la contrainte circonférentielle et la température d'essai. Aujourd'hui, il est bien établi dans les travaux de recherche que les tubes en plastique extrudés se rompent d'une manière ductile car les charges appliquées sont suffisamment hautes et la zone de rupture est caractérisée par de grandes déformations autour de la surface endommagée. Bien que la ductilité soit importante et favorable, les tubes en PE peuvent faire l'objet d'une rupture fragile quand ils sont soumis à de faibles charges pour de longues périodes de service combinées à des températures au-dessus de l'ambiante. De telles conditions favorisent habituellement le mode lent de propagation de fissure (SCG) dans des tubes en polymères.

En conséquence, les essais de charges constantes montrent deux mécanismes de propagation de fissure : une rupture ductile qui est dominée par des déformations homogènes à grande échelle dans le volume et une rupture fragile qui commence aux points de concentration de contrainte et se propage lentement, précédée par une zone de craquelures contenant de la matière endommagée. En règle générale, les courbes de défaillance sont bien décrites par la relation suivante :

$$
t_{\mathrm{f}}=C \sigma^{-n}
$$

où $C$ est une constante positive représentant le niveau de contrainte auquel le matériau se rompt à l'unité de temps (appelé aussi paramètre de capacité de charge). L'exposant $n$ est un indice d'évolution de dommage et est dans la gamme $(2,5 \leqslant n \leqslant 4,5)$ et $(20 \leqslant n \leqslant 27)$ respectivement pour les régimes fragile et ductile, alors que la contrainte de transition est égale à la moitié de la contrainte au seuil d'écoulement [7]. Dans une étude de défaillance de homopolymère de $\mathrm{PE}$ dans des conditions de contrainte plane pour une variété de températures et de vitesses, il a été constaté qu'à une température donnée, le comportement est observé comme fragile à des vitesses élevées et basses tandis que la rupture ductile caractérisait les vitesses intermédiaires. Une augmentation de la température a décalé les transitions fragile-ductilefragile à des vitesses plus élevées en raison de la concurrence entre 3 mécanismes concourants : (1) la rupture fragile par démêlage, (2) l'écoulement ductile de cisaillement et (3) la rupture fragile par scission de chaînes [8]. En utilisant l'essai d'impact de Charpy, les températures de transition fragile-ductile (BDTT) du PE ont été étudiées avec différents degrés de cristallinité. Une élévation dans la température de refroidissement de plaques en MDPE et en HDPE provoque une chute de la BDTT et une augmentation de l'énergie d'impact [9]. Avec la diminution de la densité d'enchevêtrement, le PE a montré une BDTT légèrement décroissante [10]. La rupture à court terme du PE est accomplie à travers la rupture de fibrilles dans la partie centrale de la craquelure en raison de l'écoulement global, tandis que la rupture à long terme s'est produite par des dommages fragiles d'accumulation en tant que micro-vides aux racines des fibrilles et aux joints de film [7].

La figure 1 est une représentation typique de la contrainte en fonction du temps à la rupture obtenue expérimentalement à partir d'essais de pression soutenue pour différentes résines de PE pour tube [11]. La transition de la rupture ductile à la rupture fragile est observée par le changement de pente d'une courbe donnée. Il apparaît clairement que l'extrapolation linéaire de telles données pour estimer la résistance à long terme (30 ou 50 ans) n'est pas une approche réaliste.

L'objectif de cette recherche est d'analyser la zone d'endommagement et d'étudier la transition fragileductile de fatigue (FBDT) dans des tubes de polyéthylène par corrélation entre le taux de propagation de la fissure et 


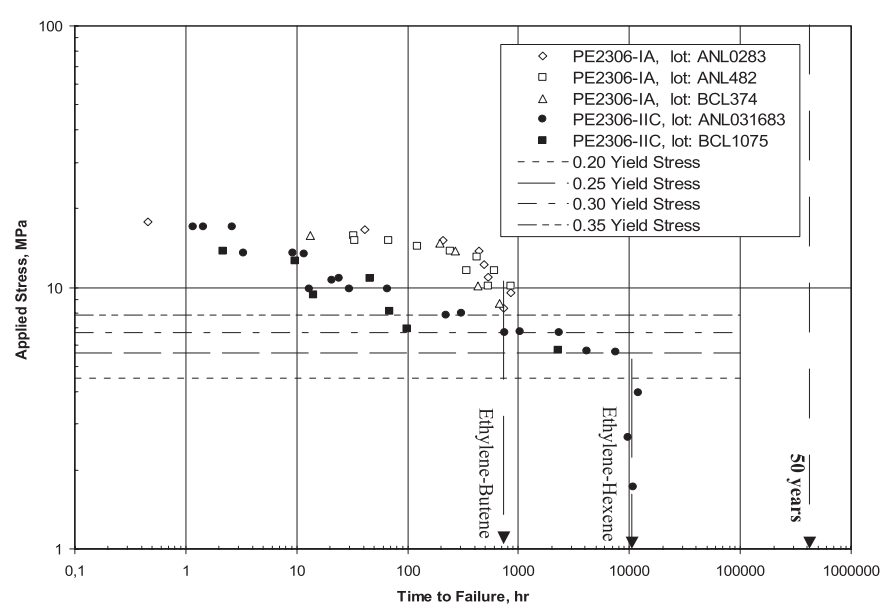

Fig. 1. Contrainte appliquée (MPa) en fonction du temps de rupture (heures) sous pression constante pour des tubes de $50 \mathrm{~mm}$ de diamètre : (PE2306-IIC : 2 lots) et (PE2306IA : 3 lots). Les lignes horizontales discontinues montrent les niveaux des essais de fatigue dans cette étude.

la quantité de travail irréversible dépensée sur les processus viscoélastiques dans le volume. Dans le but de valider cette approche caractérisant des ruptures fragile et ductile, la microscopie est employée pour analyser l'étendue de la zone d'endommagement associée à la fissure.

\section{Procédure expérimentale}

Les tubes sont extrudés d'une résine incolore (TR418) de Philips Petroleum Co. Le poids moléculaire moyen est 170000 et la densité est $939 \mathrm{~kg} \cdot \mathrm{m}^{-3}$. Le MFI est dans l'intervalle 0,4 à 1,5 et la cristallinité est de $67 \% \pm 0,03$ [12]. L'épaisseur minimale de la paroi tubulaire est de $11 \mathrm{~mm}$ et le diamètre extérieur moyen est de $115 \mathrm{~mm}$.

En raison du fait que l'état spécifique des contraintes résiduelles et les gradients micro-structuraux ont des effets importants sur le taux de propagation de la fissure, des anneaux de $28 \mathrm{~mm}$ de largeur sont obtenus pour réaliser des spécimens en forme de $C$ par usinage suivant les recommandations de ASTM E-399. La figure 2 montre le spécimen ainsi que son dispositif de maintien. Des douilles spéciales en acier ont été conçues pour renforcer les lignes de chargement du spécimen et réduire les frottements entre la goupille métallique et le polymère. La distance $S$ séparant les trous de chargement est de $25 \mathrm{~mm}$. Une entaille profonde de $2,5 \mathrm{~mm}$ a été réalisée longitudinalement sur la face interne du tube à l'aide d'une lame de rasoir et d'une presse. Des essais de propagation de fissure sous le mode traction-traction ont été effectués sur une machine servo-hydraulique MTS. La charge maximale a été choisie à $20 \%, 25 \%, 30 \%$ et $35 \%$ de $\sigma_{Y}$ tandis que le rapport de charge minimum-maximum $(R)$ a été de 0,1 afin d'éviter les effets de fluage qui se produisent à des rapports plus élevés de $R$. La fréquence a été maintenue constante à $0,5 \mathrm{~Hz}$ pour réduire l'échauffement dû à l'hystérésis. Les courbes de chargement-déchargement ont

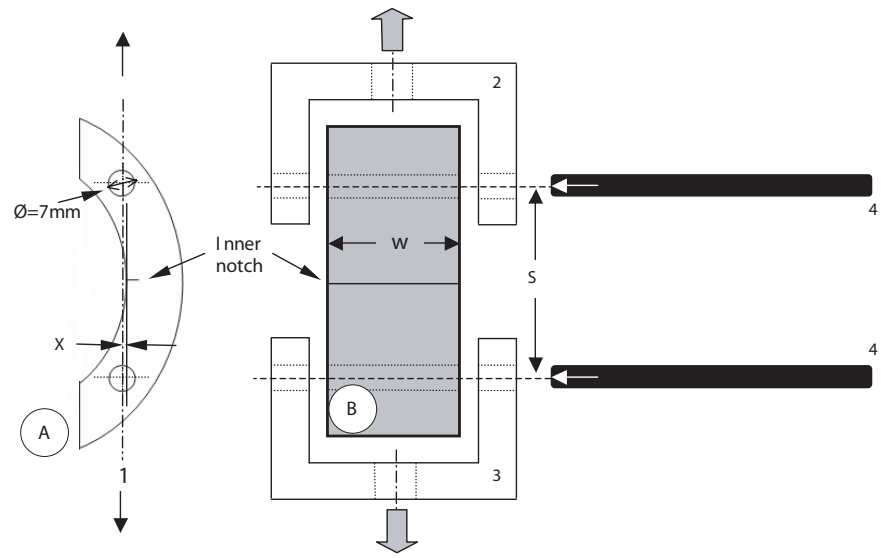

Fig. 2. Vues de droite (A) et de face (B) du spécimen en forme de $\mathrm{C}$ avec son dispositif de maintien (2 et 3 ) et les goupilles de chargement (4).

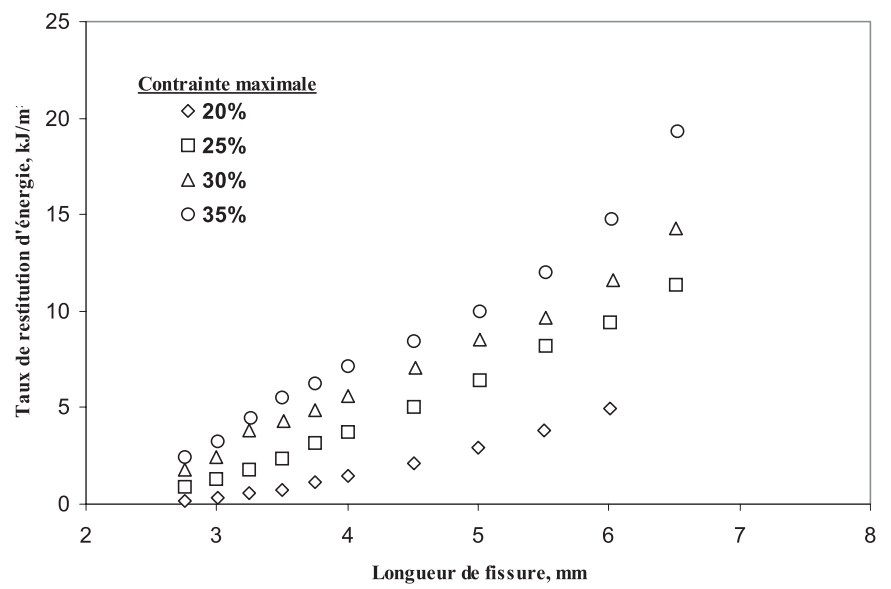

Fig. 3. Taux de restitution d'énergie à divers niveaux de chargement.

été enregistrées en fonction du déplacement à différentes longueurs de fissure.

\section{Résultats et discussion}

Il est admis que pendant la propagation d'une fissure de fatigue dans les polymères, les aspects de ductilité et de fragilité coexistent et tous deux évoluent sans interruption jusqu'à la séparation finale du matériau par déchirure. Aussi, plus les niveaux de chargement sont faibles, plus les contributions de rupture fragile sont importantes. Ceci est confirmé par les essais sous pression soutenue et également par des observations de faciès de rupture de tube en $\mathrm{PE}$ ayant eu en service. Le régime fragile est habituellement caractérisé par des stries de fatigue, qui indiquent une propagation discontinue de la fissure [5-7]. La figure 3 représente des taux de restitution d'énergie (ERR) mesurés expérimentalement à différents niveaux de charge à partir des courbes de charge-déplacement en utilisant la relation :

$$
J_{1}=-\frac{1}{t_{0}} \frac{\mathrm{d} P}{\mathrm{~d} a}
$$




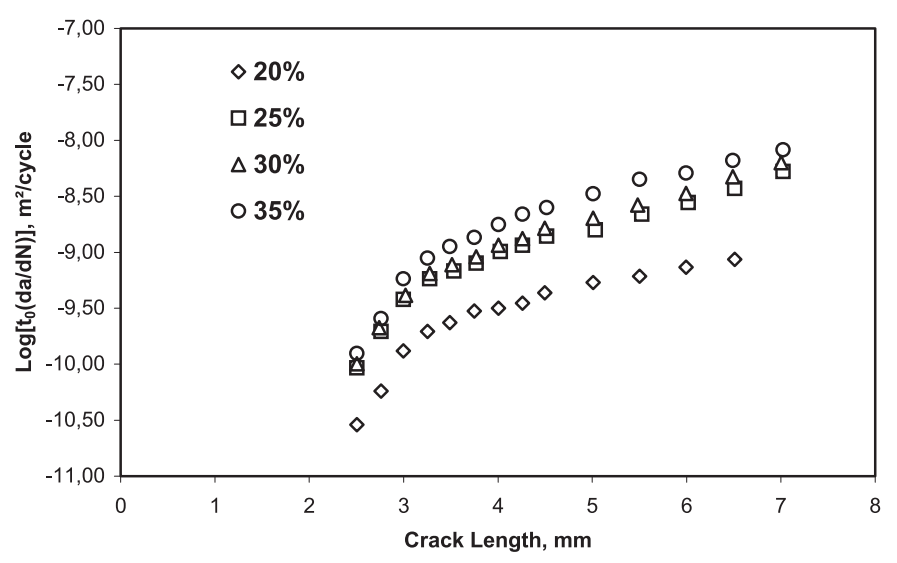

Fig. 4. Vitesse de propagation en fonction de la longueur de la fissure.

où $\left(\frac{\mathrm{d} P}{\mathrm{~d} a}\right)$ est la variation de l'énergie potentielle par variation de la longueur de fissure et $t_{0}$ l'épaisseur initiale de l'éprouvette. Le comportement des courbes ERR est semblable en variant la contrainte moyenne appliquée; une augmentation d'énergie dégagée pouvant aller jusqu'aux environs de $20 \mathrm{~kJ} . \mathrm{m}^{-2}$. Ce comportement est prévu puisque l'énergie libérée est essentiellement dépensée sur la propagation de fissure. La mesure est réalisée en utilisant des courbes de déchargement à différentes longueurs de fissure. Comparée à l'énergie élastique, cette mesure est plus représentative car elle inclut tout autre processus irréversible dû à l'incursion de la fissure. La vitesse de propagation de la fissure est illustrée dans la figure 4 en fonction de la longueur de la fissure. Puisque la rupture finale s'est produite par écoulement de matière et par déchirure, les mesures précises de la vitesse ont été faites jusqu'à une longueur de $7,5 \mathrm{~mm}$. À mesure que la contrainte maximum appliquée augmente, la vitesse de fissuration devient importante mais la contribution fragile s'amoindrit. Le comportement de la vitesse est semblable aux différents niveaux de chargement et les zones I (initiation) et II (propagation) de la fatigue sont clairement identifiables.

En utilisant les boucles d'hystérésis enregistrées à une longueur donnée de fissure, le taux de restitution d'énergie totale $J_{1}$ (Fig. 3) et la quantité de travail irréversible $W_{\mathrm{i}}$ (Fig. 5) sont déduits. L'évolution de $W_{\mathrm{i}}$ (surface comprise dans une boucle d'hystérésis) est croissante à mesure que la propagation devient prononcée et il est possible d'observer que l'accélération commence dès $3,5 \mathrm{~mm}$ de fissure.

Comme les essais normalisés par l'ASTM sont basés sur des données de fluage, il est intéressant de comparer les temps de rupture en modes de fluage et de fatigue. Une relation de proportionnalité caractérise les temps de rupture des 2 modes (Fig. 6). C'est une constatation importante qui montre que des essais de fatigue peuvent être également employés pour accélérer la rupture dans les tubes en PE et étudier la durée de vie [5,13,14].

Le tableau 1 compare les périodes d'initiation et de propagation de la fissure au nombre total de cycles. Pour le niveau $20 \%$ de $\sigma y$, l'initiation comme prévu prend plus de temps et est associée à plus de propagation fragile.

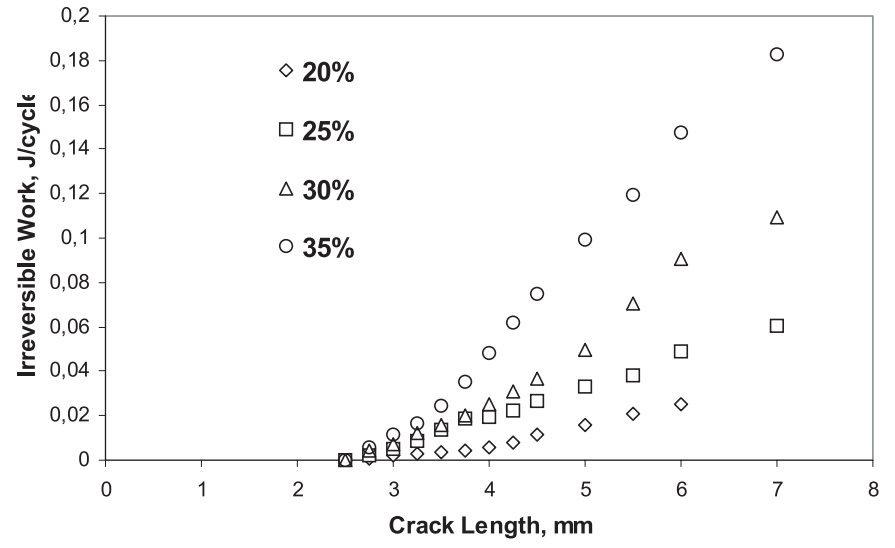

Fig. 5. Évolution du travail irréversible $\left(W_{\mathrm{i}}\right)$ en fonction de la longueur de fissure.

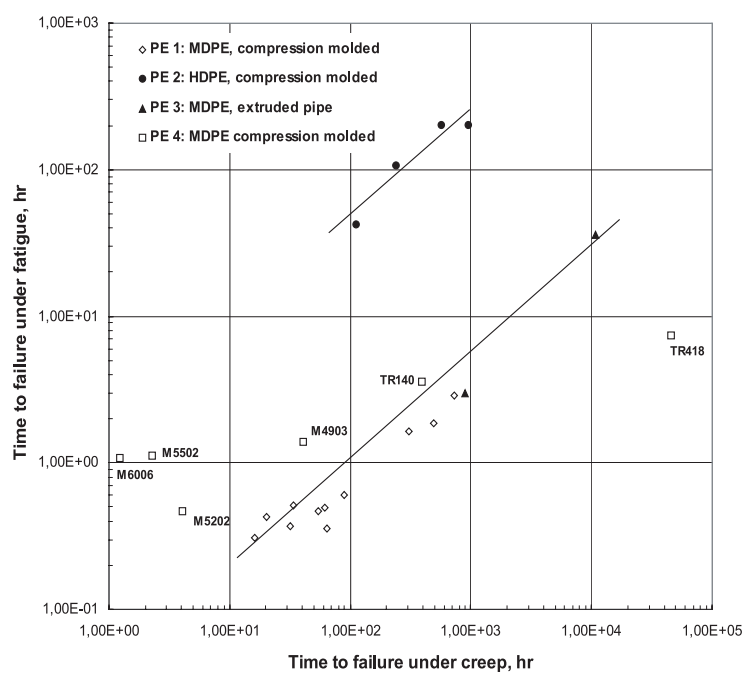

Fig. 6. Rapport entre les temps de rupture sous les modes de fluage et de fatigue pour différentes résines de polyéthylène $[5,13,14]$.

Afin d'étudier la Transition Fragile Ductile sous Fatigue (FBDT), il est intéressant d'employer l'approche énergétique proposée par Klingbeil [15] pour les matériaux ductiles endommagés sous la fatigue. Dans cette étude, la nouvelle théorie sur la propagation de fissure de fatigue, pour les solides ductiles est basée sur la dissipation de toute l'énergie plastique dans la zone d'endommagement précédant la tête de la fissure et par cycle de chargement. Le taux de propagation de fissure sous fatigue (FCG) est explicitement donné en termes de dissipation plastique d'énergie totale par cycle et de résistance à la rupture du matériau en déformation plane. Ceci est traduit par l'équation :

$$
\frac{1}{t_{0}} \frac{\mathrm{d} a}{\mathrm{~d} N}=\frac{1}{G_{\mathrm{C}}} \frac{\mathrm{d} W}{\mathrm{~d} N}
$$

où $G_{\mathrm{C}}$ est le taux de restitution d'énergie critique (J.m $\left.{ }^{-2}\right)$ et $N$ est le nombre de cycles de fatigue. 
Tableau 1. Comparaison des durées de vie d'initiation fragile et ductile.

\begin{tabular}{|c|c|c|c|c|c|}
\hline \multirow{3}{*}{ Matériau du tube } & $\% \sigma_{y}$ & $\begin{array}{c}N \text { initiation } \\
\left(10^{3} \text { cycles }\right)\end{array}$ & $\begin{array}{c}N \text { fragile } \\
\left(10^{3} \text { cycles }\right)\end{array}$ & $\begin{array}{c}N \text { ductile } \\
\left(10^{3} \text { cycles }\right)\end{array}$ & $\begin{array}{c}N \text { total } \\
\left(10^{3} \text { cycles }\right)\end{array}$ \\
\hline \multirow{4}{*}{ PE2306-IIC } & 20 & 251 & 347 & $>22 *$ & $>620 *$ \\
\cline { 2 - 6 } & 25 & 140 & 65 & 35 & 230 \\
\cline { 2 - 6 } & 30 & 90 & 50 & 66 & 206 \\
\cline { 2 - 6 } & 35 & 75 & 40 & 35 & 150 \\
\hline
\end{tabular}

* Tests arrêtés et rompus en azote liquide.
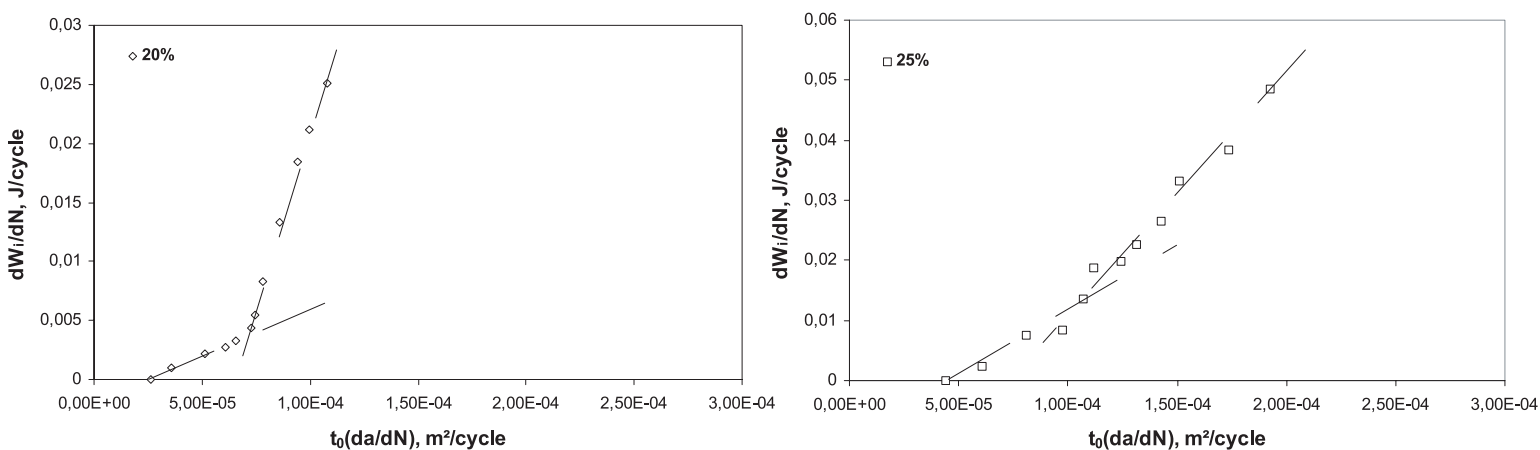

(a)

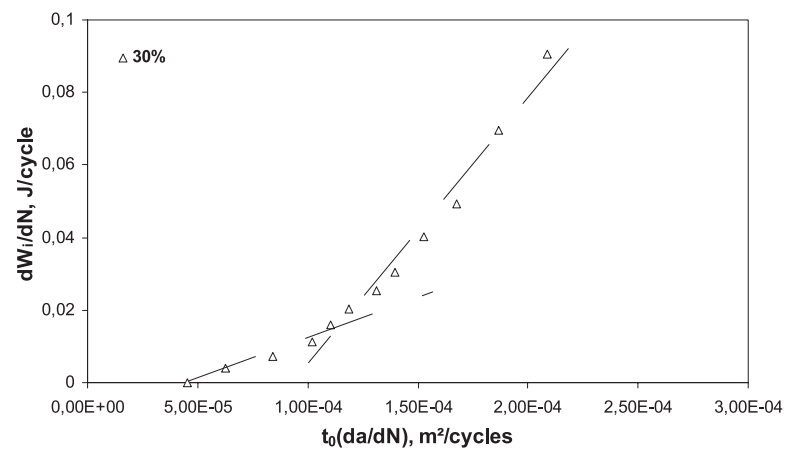

(b)

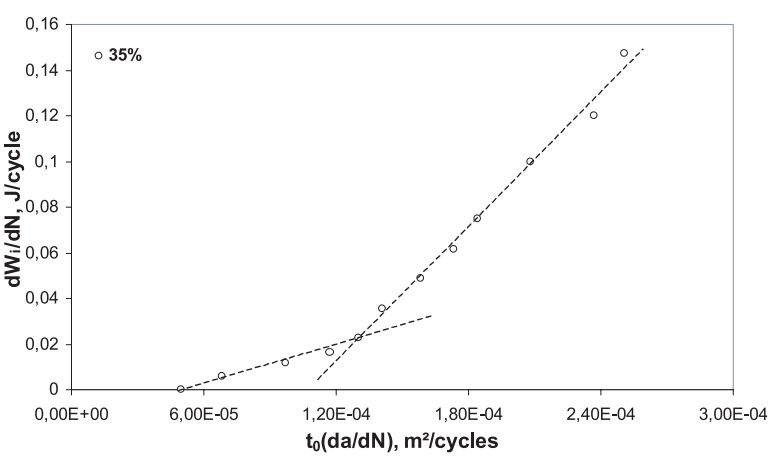

(d)

(c)

Fig. 7. Taux de travail irréversible en fonction du taux de progression de fissure à (a) $20 \%$, (b) $25 \%$, (c) $30 \%$ et (d) $35 \%$ de la contrainte appliquée.

La méthode proposée dans cette étude est basée sur la mesure de deux paramètres de fatigue : (1) le taux de progression de la fissure $\left(\mathrm{m}^{2}\right.$.cycle $\left.{ }^{-1}\right)$, obtenu à différents niveaux de charge et qui est exprimé par $\left(\frac{1}{t_{0}} \frac{\mathrm{d} a}{\mathrm{~d} N}\right)$ et (2) le taux de travail irréversible représenté par $\left(\frac{\mathrm{d} W_{i}}{\mathrm{~d} N}\right)$ qui est calculé à partir des boucles instantanées d'hystérésis à une longueur de fissure donnée. Des courbes typiques du taux de travail plastique et irréversible en fonction du taux de propagation de fissure sont construites à $20 \%$, $25 \%, 30 \%$ et $35 \%$ de la contrainte au seuil d'écoulement $\sigma_{y}$. Les résultats obtenus sont indiqués dans la figure 7 .

Les résultats indiquent que deux mécanismes de dommage sont en compétition et ils apparaissent distinctivement séparés et chacun est représenté par une pente différente. Cette observation se répète à chaque niveau de chargement représenté sur la figure 1 par les lignes pointillées horizontales. Les valeurs calculées de la ténacité donnée par l'équation (3) sont récapitulées dans le tableau 2. Les taux de restitution d'énergie critique du régime fragile se trouvent compris entre $97,7 \mathrm{~J} . \mathrm{m}^{-2}$ et $264 \mathrm{~J} \cdot \mathrm{m}^{-2}$ et ces valeurs sont plus faibles comparées aux taux d'énergie mesurés de l'évolution de l'énergie potentielle (Fig. 3). D'autre part, les énergies critiques ductiles varient de $589,5 \mathrm{~J} . \mathrm{m}^{-2}$ à $987,8 \mathrm{~J} \cdot \mathrm{m}^{-2}$. L'énergie du régime ductile est importante car elle considère beaucoup d'énergie qui est dépensée sur des dommages et les larges déformations. Il y a une évolution cohérente de $G_{\mathrm{C}}$ quand les 2 régimes (fragile et ductile) sont comparés et c'est normal que les valeurs de $G_{\mathrm{C}}$ soient fonction du niveau de chargement appliqué.

Afin de confirmer une telle approche, la microscopie est employée pour identifier la zone plastique en tête de fissure. Des sections minces ont été obtenues à partir des essais interrompus de propagation de fissure de fatigue et observées sous différentes conditions. Les résultats sont représentés dans la figure 8 . Dans le régime fragile, la 
Tableau 2. Calcul de la ténacité de la transition fragile-ductile sous la fatigue avec la détermination des coefficients de détermination $\left(\mathrm{R}^{2}\right)$.

\begin{tabular}{|c|c|c|c|c|}
\hline $\begin{array}{c}\text { Niveau de contrainte } \\
\left(\% \sigma_{y}\right)\end{array}$ & $\begin{array}{l}G_{\mathrm{C}} \text { fragile } \\
\left(\mathrm{J} . \mathrm{m}^{-2}\right)\end{array}$ & $\begin{array}{c}R^{2} \\
\text { (fragile) }\end{array}$ & $\begin{array}{c}G_{\mathrm{C}} \text { ductile } \\
\left(\mathrm{J} . \mathrm{m}^{-2}\right)\end{array}$ & $\begin{array}{c}R^{2} \\
\text { (ductile) }\end{array}$ \\
\hline 0,20 & 97,7 & 0,937 & 589,5 & 0,997 \\
\hline 0,25 & 256,5 & 0,928 & 411,6 & 0,976 \\
\hline 0,30 & 226,2 & 0,971 & 790,1 & 0,982 \\
\hline 0,35 & 264,0 & 0,980 & 987,8 & 0,988 \\
\hline
\end{tabular}
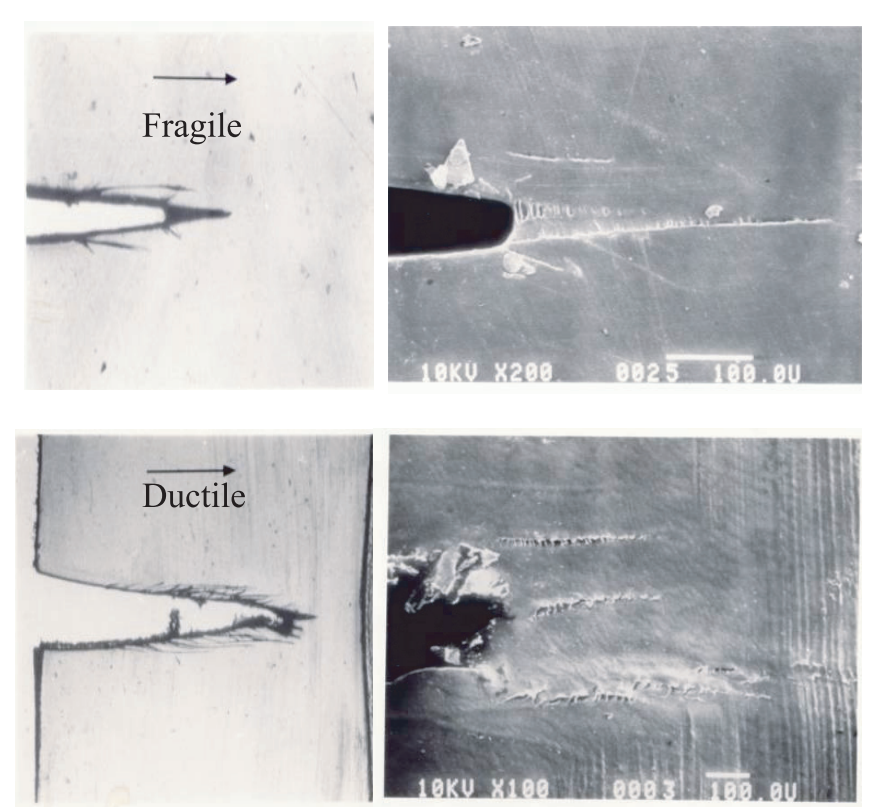

Fig. 8. Observations sur microscope optique et MEB d'une zone d'endommagement plastique en fond de fissure dans les régimes fragile et ductile.

zone plastique est très petite et est associée à une craquelure principale constituée de matériau écroui et de défauts structuraux.

Pour le régime ductile, la zone endommagée est plus importante et les craquelures sont plus longues par rapport à une fragile. L'examen au MEB illustre des craquelures multiples de part et d'autre du plan de fissure et le matériau est fortement endommagé en pointe de fissure. Autour de la zone de dommages, une autre partie du matériau transformé est fortement affectée par la plasticité pendant que ses limites s'étendent jusqu'au bord du spécimen.

Cette approche est intéressante car elle permet la séparation des contributions fragiles de celles ductiles dans un essai donné de fissuration sous fatigue du polyéthylène (FCP). L'utilisation de la microscopie permet de confirmer les étendues de chaque mécanisme à partir de l'analyse de la surface de rupture et de la diffusion du dommage dans la zone active qui représente la résistance du polyéthylène à la propagation de fissures. Comparée à la résistance hydrostatique à long terme (LTHS) définie par l'ASTM, la FCP peut être considérée comme une approche plus efficace puisqu'elle permet l'étude de la transition fragile-ductile (BDT) dans des périodes d'essai beaucoup plus courtes. Une étude récente a montré que la rupture ductile sous charges constantes, est principalement influencée par la contrainte au seuil d'écoulement du PE. L'examen des données de ruptures ductiles aux différentes températures a également indiqué qu'il y a une amélioration de la performance avec la température ce qui pourrait être lié à la relaxation progressive des efforts internes tel que préconisé par [16].

\section{Conclusions}

L'accélération de la propagation de fissure peut être obtenue en utilisant des chargements de fatigue avec la fréquence appropriée pour éviter le chauffage dû à l'hystérésis du polyéthylène. La rupture fragile peut aussi être reproduite dans l'essai de fatigue et plus la contrainte moyenne appliquée est faible, plus la contribution fragile est importante. Quant à la transition fragile-ductile de fatigue (FBDT) dans les matériaux semi-cristallins comme le PE, il est possible de l'analyser en utilisant le travail irréversible et le taux de propagation de fissure pour obtenir le taux de restitution d'énergie critique pour les mécanismes de dommages présents de manière séparée. Comparé au taux de restitution d'énergie obtenu à partir des mesures d'énergie potentielle, le taux d'énergie critique calculé est inférieur d'un ordre de grandeur. Pour les charges maximales entre $20 \%$ et $35 \%$ de la contrainte de traction au seuil d'écoulement, les résultats montrent des

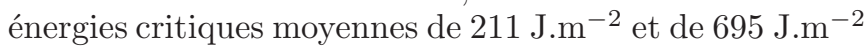
respectivement pour des régimes fragile et ductile.

\section{Références}

[1] A. Chudnovsky, Y. Sulkhin, Application of crack layer theory to modelling of slow crack growth in polyethylene, Int. J. Fract. 97 (1999) 83-102

[2] L. Hubert, L. David, R. Séguéla, G. Vigier, C. CorfiasZuccalli, Y. Germain, Physical and mechanical properties of PE pipes in relation to molecular architecture: Shortterm creep of isotropic and drawn materials, J. Appl. Polym. Sci. 84 (2002) 2308-2317

[3] H.B.H. Hamouda, M. Simoes-Betbeder, R. Grillon, P. Blouet, N. Billon, R. Piques, Creep damage mechanisms in polyethylene gas pipes, Polymer 42 (2001) 5425-5437

[4] K. Chaoui, A. Moet, A Chudnovsky, Consequences of residual stress on crack propagation in PE Pipes, Proc. 
10th Inter. Conf. Experim. Mech., Lisbon, Portugal, 1994 pp. $811-816$

[5] Y. Zhou, X. Lu, Z. Zhou, B. Brown, The relative influence of molecular structure on brittle fracture by fatigue and under constant loads in polyethylene, Poly. Eng. and Sci. 36 (1996) 2101-2107

[6] B.-H. Choi, Z. Zhou, A. Chudnovsky, S.S. Stivala, K. Sehanobish, Bosnyak C. P., Fracture initiation associated with chemical degradation: observation and modelling, Int. J. Solid. Struct. 42 (2005) 681-695

[7] D.-M. Duan, J.G. Williams, Craze testing for tough polyethylen, J. Mater. Sci. 33 (1998) 625-638

[8] Kitao K., A study of brittle-ductile transition in polyethylene, Polym. Eng. Sci. 37 (1997) 777-788

[9] A. Paizis, P. Leevers, Orientation and strain cycle effects on the impact performance of polyethylene, Polym. Eng. Sci. 45 (2005) 596-605

[10] K. Kitao, Effect of entanglement on brittle-ductile transition in polyethylene, Polym. Eng. Sci. 41 (2001) 11461155
[11] J.E. Young, L.A. Raphaelian, D.T. Raske, GRI Project Review Meeting, Chicago, USA, 1986

[12] J.M. Crissman, Reference Standard Polyethylene Resins and Piping Materials, GRI Final Report 87-0326, Chicago, Illinois, USA, 1987

[13] K. Kadota, S. Chum, A. Chudnovsky, Bridging the PE lifetime under fatigue and creep conditions with its crystallization behaviour, J. Appl. Polym. Sci. 49 (1999) 863-875

[14] M. Parsons, E.V. Stepanov, A. Hiltner, B. Baer, Correlation of stepwise fatigue and creep slow crack growth in high-density polyethylene, J. Mater. Sci. 35 (2000) 1857-1866

[15] N.W. Klingbeil, A total dissipated energy theory of fatigue crack growth in ductile solids, Int. J. Fract. 25 (2003) 117-128

[16] R.K. Krishnaswamy, Analysis of ductile and brittle failures from creep rupture testing of high-density polyethylene (HDPE) pipes, Polymer 46 (2005) 11664-1167 